\title{
Search for the gravitational wave memory effect with the Parkes Pulsar Timing Array
}

\author{
Jingbo Wang ${ }^{1,2,3}$, G. Hobbs ${ }^{3}$ and Na Wang ${ }^{1,4}$ \\ ${ }^{1}$ Xinjiang Astronomical Observatory, Chinese Academy of Science, \\ 150 Science 1-Street, Urumqi, Xinjiang, China, 830011 \\ email: wangjingbo@xao.ac.cn \\ ${ }^{2}$ University of the Chinese Academy of Sciences, No.19A Yuquan Road, \\ Beijing, China, 100049 \\ ${ }^{3}$ CSIRO Astronomy and Space Science, Australia Telescope National Facility, \\ PO Box 76, Epping, NSW 1710, Australia \\ ${ }^{4}$ Key Laboratory of Radio Astronomy, Chinese Academy of Science, \\ Nanjing, China, 210008
}

\begin{abstract}
Gravitational wave bursts produced by supermassive binary black hole mergers will leave a persistent imprint on the space-time metric. Such gravitational wave memory signals are detectable by pulsar timing arrays as a glitch event that would seem to occur simultaneously for all pulsars. In this paper, we describe an initial algorithm which can be used to search for gravitational wave memory signals. We apply this algorithm to the Parkes Pulsar Timing Array data set. No significant gravitational wave memory signal is founded in the data set.
\end{abstract}

Keywords. gravitational waves, pulsar, black holes.

\section{Introduction}

It is believed that observations of MSPs will lead to the direct detection of gravitational waves (GWs) with frequencies of $10^{-9}-10^{-7} \mathrm{~Hz}$ (Hobbs et al. 2005, Jenet et al. 2005). Many observing projects have now been started with the goals of observing a large enough sample pulsars with sufficient precision to detect GW signals. Such projects are known as pulsar timing arrays (PTAs, Foster 1990). Here we make use of data set from the Parkes Pulsar Timing Array project (PPTA; Manchester et al. 2012). The Parkes observations have already been used in searching for the GW emission from individual, non-evolving, supermassive black hole binaries (Yardley et al. 2010), placing an upper limit on a background of GWs (Jenet et al. 2006) and in attempting to detect such a GW background (Yardley et al. 2011).

In contrast to earlier searches for GWs using the PPTA data sets, in this paper, we focus on the GW memory (GWM) phenomenon. The expected source is a supermassive binary black hole (SMBBH) system that has coalesced (Favata 2009). At the coalescence stage of the SMBBH, a permanent change in the space-time metric will be induced. Cordes \& Jenet (2012) and van Haasteren \& Levin (2010) have previously shown that pulsar timing arrays are sensitive to such GW memory events. When such a GW signal passes a pulsar or the Earth, it will lead to a simple frequency jump in the observed pulse frequency of the pulsar. The timing residuals will have the characteristics of a simple glitch event. GW memory events passing a single pulsar will lead to a glitch-like event in the timing residuals of that pulsar only. GW memory events passing the Earth will lead to a glitch-like event seen in the timing residuals of all pulsars with the size of the pulse frequency jump depending upon the GW source-Earth-pulsar angle. The pre-fit 
timing residuals induced by the GWM signal that occurred at $t=t_{0}$ can be written as:

$$
r(t)_{\text {prefit }}=\frac{1}{2} h^{\mathrm{mem}}(1-\cos \theta) \cos 2 \phi\left(t-t_{0}\right) \Theta\left(t-t_{0}\right),
$$

In the above, $\theta$ is the GW source-Earth-pulsar angle, $\phi$ is the angle between the wave's principle polarization and the projection of the pulsar onto the plane perpendicular to the propagation direction, and $\mathrm{h}^{\mathrm{mem}}$ is the amplitude of the GWM signal. Therefore, the pre-fit timing residuals induced by the Earth term of GWM signal will give rise to a linear increase of the pre-fit residuals with time.

\section{Method}

Here we describe our current algorithm and present initial results. The completed algorithm and our final results will be published elsewhere. We have updated the TEMPO2 pulsar timing model to include the effect of a GWM event. The position of pulsars and the GW source are specified in the equatorial coordinate system by their right ascension and declination $(\alpha, \delta)$. The principle polarisation of GW is defined in a coordinate system $\left(\mathrm{r}_{g}, \alpha_{g}, \delta_{g}\right)$ where the GW propagates along the $-r_{g}$ direction (See Fig. 1 of Hobbs et al. 2009). Since TEMPO2 only implements a linear least-squares-fitting procedure for improving the pulsar timing model, TEMPO2 can only be used to fit for the amplitude of the GWM event. If, as usual, the position, epoch and/or polarisation angle is unknown and it is necessary to determine these parameters using a different procedure. A global fitting algorithm (first described in Champion et al. 2010) is used to fit for pulse, astrometric and orbital parameters of each pulsars individually whilst simultaneously fitting for $h^{\mathrm{mem}}$. In order to account for the unknown polarisation angle (PA) we carry out two fits, one with $P A=0$ and the second with $P A=\pi / 4$. For each fit we obtain a measurement of $h^{\mathrm{mem}}\left(\mathrm{h}_{1}\right.$ and $\mathrm{h}_{2}$ for $P A=0$ and $P A=\pi / 4$, respectively) and their corresponding uncertainties ( $\sigma_{1}$ and $\sigma_{2}$ respectively). Then we form the following detection statistic:

$$
S=\left(\frac{h_{1}}{\sigma_{1}}\right)^{2}+\left(\frac{h_{2}}{\sigma_{2}}\right)^{2}
$$

Coles et al. (2011) discussed the issues arising from fitting a pulsar timing model in the presence of non-white noise. For this work, we obtain a simple analytic model of the red noise for each pulsar (as described in Manchester et al. 2012) and use the generalised least-squares-fitting routines (often referred to as "Cholesky" fitting) within TEMPO2.

\section{Observations}

We make use of the Parkes Pulsar Timing Array (PPTA) data set which is described in Manchester et al. (2012). These data include regular observations of 20 millisecond pulsars at intervals of 2-3 weeks from 2005 to 2011. All observations were taken with the Parkes 64-m radio telescope. The typical integration time for each pulsar is about $1 \mathrm{hr}$. Most of the timing offsets between the different observing systems have been removed. However, some of the arbitrary jumps from the timing model included in the Verbiest et al. (2008, 2009) were retained (Manchester et al. 2012). Variations of dispersion measure were corrected by using multi-frequency observations. Timing residuals were formed using the TEMPO2 software package (Hobbs et al. 2006) making use of the JPL DE421 Solar System ephemeris (Folkner et al. 2008) and refered to terrestrial time as realised by BIPM2011. 


\section{Results}

We searched for a GWM signal using the algorithm described above. The measured statistic value ranges from 0 to 20 for different trial positions and glitch epochs. By comparison, the largest detection statistic value is $\sim 126$ after a small simulated GWM signal is added into the PPTA data set. We therefore conclude that there is not a large GWM signal in the PPTA data set. We are now carrying out statistical tests of the algorithm in order to answer the following questions: "what is the largest GWM signal that could be present in our data?" and "what is the probability that we have already made a detection of a small GWM signal?". These statistical tests are not yet complete, but are based on Monte-Carlo simulations in which we inject small GWM signals into simulated data sets and measure the detection statistic values.

It is unlikely that we will make a detection of a GWM event with the existing Parkes data set. However, the data sets continue to get longer and future analyses are likely to be carried out with a combination of data from different observatories Worldwide. In the longer term, it is expected that future telescopes such as FAST, Urumqi 110-m and the SKA Phase I will provide data sets in which GWM events will be easily detectable.

\section{Acknowledgments}

This work is supported by National Basic Research Program of China (973 Program 2009CB824800 and 2012CB821800), NSFC project (No. 11173041, No. 11173042 and No. 11203063), the Knowledge Innovation Program of the Chinese Academy of Sciences, Grant No. KJCX2-YW-T09, West Light Foundation of CAS (No. XBBS201021 and No. XBBS201123) and Natural Science Foundation of Xinjiang, China Grant No.Y1000201. GH acknowledges support from the Australian Research Council \#DP0878388.

\section{References}

Champion, D. J., Hobbs, G. B., Manchester, R. N., et al. 2010, ApJ, 720, L201

Coles, W., Hobbs, G., Champion, D. J., Manchester, R. N., \& Verbiest, J. P. W. 2011, MNRAS, 418,561

Cordes, J. M. \& Jenet, F. A. 2012, ApJ, 752, 54

Favata M., 2009, PRD, 80, 024002

Foster R. S., Backer D. C. 1990, ApJ, 361, 300

Hobbs G. 2005, PASA, 22, 179

Hobbs, G. B., Edwards, R. T., \& Manchester, R. N. 2006, MNRAS, 369, 655

Hobbs, G., Jenet, F., Lee, K. J., et al. 2009, MNRAS, 394, 1945

Jenet F., Hobbs G., Lee K., Manchester R. 2005, ApJ, 625, L123

Jenet, F. A., Hobbs, G. B., van Straten, W., et al. 2006, ApJ 653, 1571

Manchester R., Hobbs, G. B., Bailes,M., et al. 2012, PASA, submitted van Haasteren R., Levin Y., McDonald P., Lu T., 2009, MNRAS 395, 1005

Verbiest, J. P. W., Bailes, M., van Straten, W., et al. 2008, ApJ, 679, 675

Verbiest J. P. W.., Bailes, M., Coles, W. A. et al. 2009, MNRAS 400, 951

Yardley, D. R. B., Coles, W. A., Hobbs, G. B., et al. 2011, MNRAS 414, 1777

Yardley, D. R. B., Hobbs, G. B., Jenet, F. A., et al. 2010, MNRAS, 407, 669

Folkner, W. M., Williams, J. G., \& Boggs, D. H. 2008, The Planetary and Lunar Ephemeris DE 421, JPL IOM 343R-08-003 